\title{
Study on Flow and Heat Transfer Characteristics of Supercritical Kerosene in a Round Tube
}

\author{
Feng Gao, Qian Zhang a , Hongyu Xiao, Fengli Chen, Xuefeng Xia \\ Air and Missile Defense College, Air Force Engineering University, 710051 Shaanxi Xian, China
}

\begin{abstract}
The finite volume discrete solution of the Navier-Stokes equation and the RNG model of the turbulence model are used to numerically simulate the flow and heat transfer characteristics of supercritical kerosene in a circular tube. The results show that as the inlet mass flow increases, the wall surface temperature and the central flow oil temperature gradually decrease, and the pressure loss becomes larger. As the inlet temperature increases, the wall surface temperature and the central flow oil temperature both increase. When the heat flux density is constant, as the pressure increases, the deterioration of heat transfer will be weakened, and increasing the pressure can improve the effect of convection heat transfer.
\end{abstract}

\section{Introduction}

Hydrocarbon fuel, as a new type of engine fuel, has advantages that other fuels do not have, making its research very important for the development of superb technology. And studying the heat transfer flow of hydrocarbon fuel in the cooling passage of supercombustion stamping engine is helpful to understand the flow and heat transfer phenomenon and mechanism of hydrocarbon fuel in the passage. For the structure of cooling passage of super-combustion stamping engine and the entire engine, Design helps.

As the flying Mach number increases, the combustion is often in a supersonic state, which requires higher and higher fuel transportation speeds, which causes the pressure of the fuel to rise above its critical pressure, and the fuel absorbs heat in the cooling channel. Its own temperature is also rising. When it reaches the critical temperature of the pressure, it becomes a supercritical fluid. Compared with pure materials such as $\mathrm{H} 2 \mathrm{O}$ and $\mathrm{CO} 2$, the flow and heat transfer of hydrocarbon fuels under supercritical pressure have similarities and have their own complex characteristics. Therefore, it is very important to study the flow and heat transfer characteristics of hydrocarbon fuel in the supercritical state.

The previous researches on inorganic working fluids and pure substances are more. However, most of the supercombustion stamping engine propellants use aviation kerosene, and there is less research on them. Kerosene is a complex mixture of various organic substances. The heat transfer flow in the channel is more complicated, and its research on the heat transfer characteristics in the cooling channel is of great significance.

This article simulates the flow and heat transfer characteristics of kerosene in a slender circular tube, analyzes the mechanism of the deterioration of heat transfer, and studies the heat transfer flow of kerosene under different inlet conditions.

\section{Calculation models and numerical methods}

\subsection{Physical model and geometry}

The research object is a two-dimensional axisymmetric round pipe with a diameter of $12.0 \mathrm{~mm}$ and a length of $5.5 \mathrm{~m}$. The first section is $1.0 \mathrm{~m}$ long without heating. It is guaranteed that the kerosene entering the heating section is a fully developed tube flow, and the heating section is 4.5 m. Figure 1 shows the calculation area and related parameters of the circular tube. The kerosene flows through the horizontal tube from left to right. Because it is only to explore the heat transfer and flow characteristics of supercritical kerosene in the tube, and the diameter of the round tube being studied is small, the effect of buoyancy is not considered in the calculation. Therefore, the numerical simulation can be simplified to an axisymmetric two-

\footnotetext{
a Corresponding author: zhangqian8913@163.com
} 
dimensional plane. It greatly reduces the calculation amount and saves the calculation time ${ }^{[1]}$.

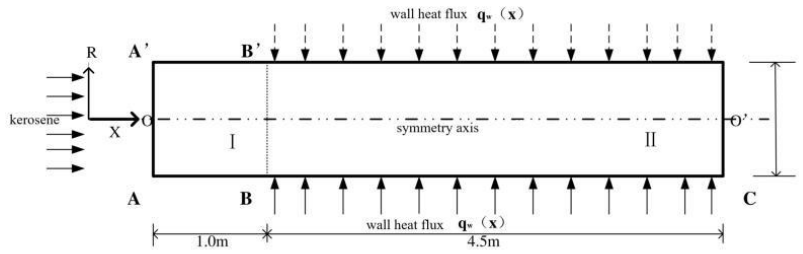

Figure 1. Physical model diagram.

\subsection{Boundary conditions}

The $1.0 \mathrm{~m}$ inlet section is not heated, so that the kerosene entering the heating section can fully develop tube flow. The inlet $\mathrm{AO}$ is the boundary condition for the mass flow into the inlet, mass flow $\dot{m}=\dot{m}_{0}$, inlet pressure $p=p_{0}$, inlet temperature $T=T_{0}$, turbulent kinetic energy $k=k_{0}$, and turbulent dissipation rate $\varepsilon=\varepsilon_{0}$, where $k_{0}$ and $\varepsilon_{0}$ are shown by equations (1) and (2) respectively.

$$
\begin{gathered}
k_{0}=\frac{3}{2}\left[\bar{u}\left(0.16 R e_{D_{H}}\right)^{1 / 8}\right]^{2} \\
\varepsilon_{0}=C_{u}{ }^{3 / 4} \frac{k^{3 / 2}}{l}
\end{gathered}
$$

Among them, $C_{u}$ is the empirical constant (about 0.09); $\bar{u}$ is the average flow velocity; $\operatorname{Re}_{D_{H}}$ is the Reynolds

$$
\begin{aligned}
& \frac{\partial}{\partial x}\left(\rho u_{x} u_{x}\right)+\frac{\partial}{\partial y}\left(\rho u_{y} u_{x}\right)=\frac{\partial}{\partial x}\left(\mu \frac{\partial u_{x}}{\partial x}\right)+\frac{\partial}{\partial y}\left(\mu \frac{\partial u_{x}}{\partial y}\right)-\frac{\partial p}{\partial x}+S_{u_{x}} \\
& \frac{\partial}{\partial x}\left(\rho u_{y} u_{x}\right)+\frac{\partial}{\partial y}\left(\rho u_{y} u_{y}\right)=\frac{\partial}{\partial x}\left(\mu \frac{\partial u_{y}}{\partial x}\right)+\frac{\partial}{\partial y}\left(\mu \frac{\partial u_{y}}{\partial y}\right)-\frac{\partial p}{\partial y}+S_{u_{y}}
\end{aligned}
$$

Energy conservation equation:

$$
\frac{\partial}{\partial x}\left(\rho u_{x} T\right)+\frac{\partial}{\partial y}\left(\rho u_{y} T\right)=\frac{\partial}{\partial x}\left(\frac{k}{c_{p}} \frac{\partial T}{\partial x}\right)+\frac{\partial}{\partial y}\left(\frac{k}{c_{p}} \frac{\partial T}{\partial y}\right)+S_{T}
$$

The turbulence model uses a $R N G k-\varepsilon$ model, and its governing equation is:

$$
\begin{aligned}
& \frac{\partial}{\partial x_{i}}\left(\rho k u_{i}\right)=\frac{\partial}{\partial x_{j}}\left[\alpha_{k} \mu_{e f f} \frac{\partial k}{\partial x_{j}}\right]+G_{k}+\rho \varepsilon \\
& \frac{\partial}{\partial x_{i}}\left(\rho \varepsilon u_{i}\right)=\frac{\partial}{\partial x_{j}}\left[\alpha_{\varepsilon} \mu_{e f f} \frac{\partial \varepsilon}{\partial x_{j}}\right]+\frac{C_{1 \varepsilon}^{*}}{k} G_{k}-C_{2 \varepsilon} \rho \frac{\varepsilon^{2}}{k}
\end{aligned}
$$

Among them, 


$$
\begin{aligned}
& \mu_{e f f}=\mu+\mu_{t} \\
& \mu_{t}=\rho C_{\mu} \frac{k^{2}}{\varepsilon} \\
& C_{\mu}=0.0845, \alpha_{k}=\alpha_{\varepsilon}=1.39 \\
& C_{1 \varepsilon}^{*}=C_{1 \varepsilon}-\frac{\eta\left(1-\eta / \eta_{0}\right)}{1+\beta \eta^{3}} \\
& C_{1 \varepsilon}=1.42, C_{2 \varepsilon}=1.68 \\
& \eta=\left(2 E_{i j} \cdot E_{i j}\right)^{1 / 2} \frac{k}{\varepsilon} \\
& E_{i j}=\frac{1}{2}\left(\frac{\partial u_{i}}{\partial x_{j}}+\frac{\partial u_{j}}{\partial x_{i}}\right) \\
& \eta_{0}=4.377, \beta=0.012
\end{aligned}
$$

\subsection{Numerical calculation method}

In this paper, finite volume discrete solution is used for the Navier-Stokes equation, and the $\mathrm{RNG} k-\varepsilon$ model is used for the turbulence model. Gambit software was used to mesh the cooling channels. In order to improve the calculation accuracy, the mesh in the near-wall region was encrypted and the encryption of the near-wall mesh was guaranteed to be in line with $\mathrm{y}^{+} \leqslant 1$.

The governing equation is solved according to incompressible and variable physical conditions. The speed and pressure coupling uses the SIMPLEC algorithm, and the time advancement uses the implicit Gauss-Seidel method; the convection term and the diffusivity adopt the upwind difference format. The first-order upwind accuracy is used for calculation, and the second-order accuracy is used when the convergence is fast. All variables in the governing equation have second-order accuracy in time and space. User-defined wall heat flux density and kerosene physical property parameters. The physical property parameters of kerosene in supercritical state have been given in the literature ${ }^{[6-10]}$. The kerosene at different pressures and temperatures calculated by Supertrapp developed by the National Institute of Technology and Standards (NIST) In the calculation of physical properties, the method of data fitting is used.

\subsection{Model reliability verification}

In order to verify the reliability of the numerical model, we need to compare the numerical calculation results with experimental data. The calculation results in this paper are compared with the experimental data given in ${ }^{[2]}$ to verify the numerical method.

Figure 2 shows the comparison of the calculated wall temperature and oil temperature distribution of the tube with the experimental data. It can be seen from Figure 2 that the difference between the numerical results and the experimental data is less than $10 \mathrm{~K}$, which indicates that the numerical calculation can simulate the flow and heat transfer of kerosene under supercritical conditions, and has high calculation accuracy.

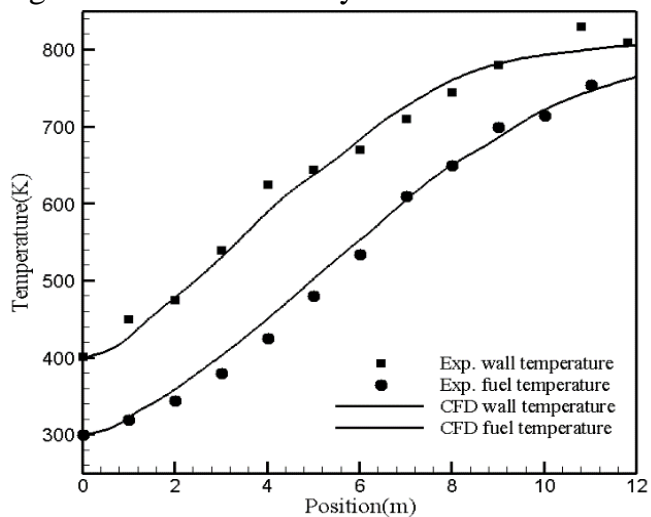

Figure 2. Comparison with experimental data.

\section{Calculation results and analysis}

\subsection{Heat transfer characteristics at different mass flow rates}

In order to study the heat transfer of aviation kerosene in a circular tube at different mass flow rates, the inlet mass flow rate was changed from $0.06 \mathrm{~kg} / \mathrm{s}$ to $0.12 \mathrm{~kg} / \mathrm{s}$ at an inlet pressure of $4 \mathrm{MPa}$, an inlet temperature of $300 \mathrm{~K}$, and a heat flux density distribution along the axial direction as shown in Figure 3,to obtain the distribution of the inner wall surface temperature and the central flow temperature along the flow direction, as shown in FIG. 3.

Figure $3 \mathrm{a}$ shows the distribution of wall temperature changes under different mass flows. It can be seen that each curve shows a trend of increasing first and then decreasing. As the mass flow increases, the wall temperature gradually decreases. When each temperature curve decreases, The corresponding position in the $\mathrm{x}$ direction also gradually moves toward the exit direction. Figure $3 \mathrm{~b}$ shows the change of the central flow temperature. It can be seen that the central flow temperature gradually increases with the axial direction. The larger mass flow corresponds to the lower central flow oil temperature. The observed mass flow is $0.08 \mathrm{~kg} / \mathrm{s}$ and $0.10 \mathrm{~kg} / \mathrm{s}$. It can be seen from the two curves of that when $\mathrm{x}=4.2 \mathrm{~m}$ and $\mathrm{x}=$ $5 \mathrm{~m}$, the change trend of the central flow oil temperature has changed, and the temperature rise trend has become sharp. At this time, the corresponding wall temperature curve is exactly At the position just to be lowered, it can be known from the analysis in Section 3.2 that the reason for the change in the central flow oil temperature is that the heat transfer of supercritical kerosene at this time is just at the stage of heat transfer enhancement, and the wall surface transfers The heat increases, so the wall temperature starts to drop and the oil temperature starts to rise. When the mass flow rate is $0.12 \mathrm{~kg} / \mathrm{s}$, the temperature of kerosene can hardly reach the pseudo-critical temperature, so this trend is not obvious. When the mass of kerosene is $0.06 \mathrm{~kg}$ / s, the temperature of kerosene is too high at this time, because kerosene will have obvious thermal cracking reaction at excessively high temperature (greater than $800 \mathrm{~K})$. The studies were performed in the temperature range below $800 \mathrm{~K}$. 


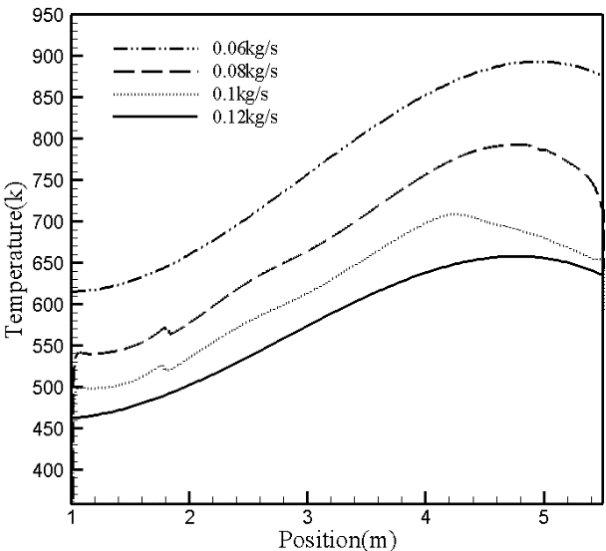

a. Wall temperature distribution along the axis

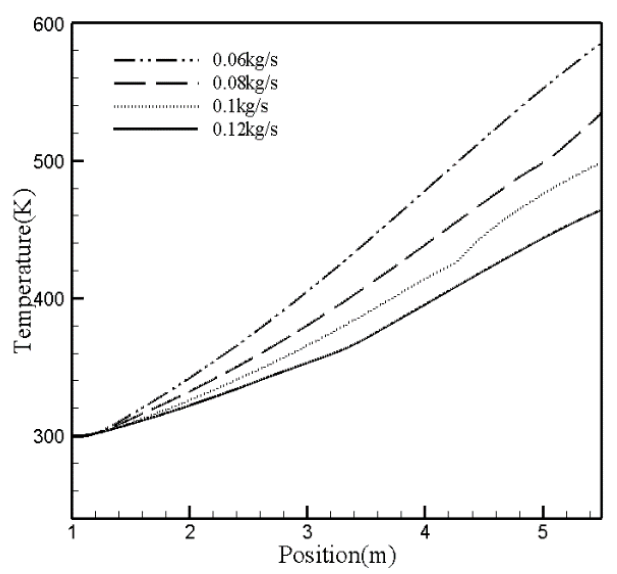

b.Center flow oil temperature distribution along the axis

Figure 3. Temperature distribution of inner wall surface and central flow oil temperature in the axial direction.

Figure 4 shows the oil pressure distribution at the central flow of different mass flow, it can be seen that the kerosene pressure along the channel gradually decreases, with the increase of the mass flow, kerosene pressure gradually increases, the corresponding pressure loss is gradually increased, which is mainly due to the increase of mass flow flow, resulting in the increase of flow rate, the flow resistance along the process increases, thus loss of kinetic energy, increased pressure loss.

Figure 5 shows the change of convective heat exchange coefficient with the wall temperature under different mass flows. As can be seen from the figure, the change trend of convection heat exchange coefficient is basically the same in the case of four mass inlets. The initial stage of heating begins to increase with the increase of temperature convection heat exchange coefficient, which is characterized by normal heat transfer phenomenon, and with the further increase of temperature, when the wall temperature reaches near the critical temperature of kerosene, the convective heat exchange coefficient begins to fall back and the heat transfer deterioration occurs, and then the heat exchange coefficient increases again, which manifests itself as the phenomenon of heat transfer reinforcement. The wall temperature change smaller in the corresponding heat transfer deterioration, both in the $650 \mathrm{~K}-720 \mathrm{~K}$ range, near the proposed critical temperature of kerosene, and the wall temperature change of secondary heat transfer reinforcement is also small, between $670 \mathrm{~K}$ $770 \mathrm{~K}$, slightly higher than the proposed critical temperature of kerosene, and when the mass flow is large, the temperature of the heat transfer deterioration and reheat heat transfer is lower.

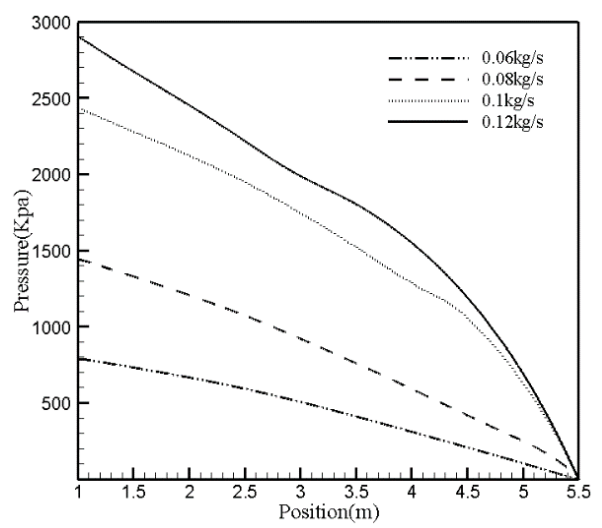

Figure 4. Oil pressure distribution at central flow

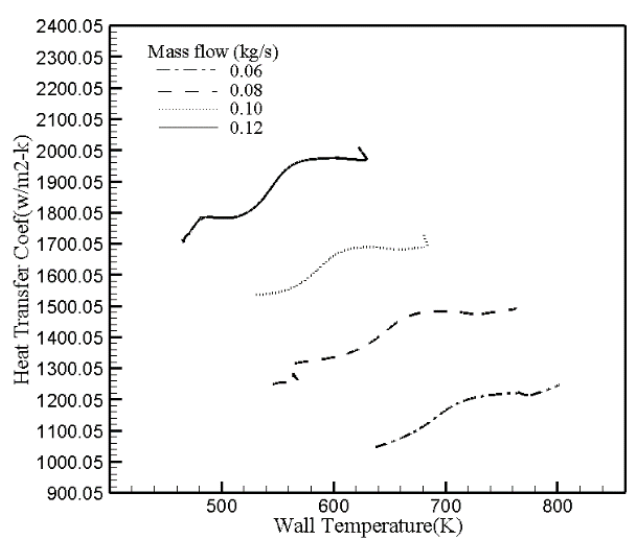

Figure5. Change of convection heat transfer coefficient at different mass

flows.

with wall temperature at different mass flow rates.

\subsection{Heat transfer characteristics at different inlet temperatures}

In order to study the heat transfer of aviation kerosene in a circular tube at different inlet temperatures, the inlet temperature was set at the inlet pressure of $4 \mathrm{MPa}$, the inlet mass flow rate of $0.1 \mathrm{~kg} / \mathrm{s}$, and the heat flux density was distributed axially as shown in Figure 3, Change from 200K to $600 \mathrm{~K}$. The specific entry parameters are shown in Table 1

It can be seen from the table that as the temperature of the inlet changes, the density of kerosene and the Reynolds number of the inlet change greatly. Specifically, as the temperature increases, the density decreases, the Reynolds number increases, and the Reynolds number changes greatly, increasing from 4368. As large as 68140.8, an increase of an order of magnitude.

Table 1. Various operating conditions at different inlet temperatures

\begin{tabular}{c|c|c|c|c}
\hline $\mathrm{P}_{0}(\mathrm{MPa})$ & $\mathrm{T}_{0}(\mathrm{~K})$ & $\dot{m}(\mathrm{~kg} / \mathrm{s})$ & $\rho_{0}\left(\mathrm{~kg} / \mathrm{m}^{3}\right)$ & $R e_{0}$ \\
\hline 4 & 200 & 0.1 & 780 & 4368 \\
4 & 300 & 0.1 & 791.56 & 8922.35 \\
4 & 400 & 0.1 & 703.09 & 20906.67 \\
4 & 500 & 0.1 & 611.80 & 39104 \\
4 & 600 & 0.1 & 507.33 & 68140.8 \\
\hline
\end{tabular}

Figure 6 shows the changes in wall temperature and central 
flow oil temperature along the axial direction at different inlet temperatures. As can be seen from the figure, both the wall surface temperature and the central flow oil temperature increase as the inlet temperature increases. When the inlet temperature is $200 \mathrm{~K}$, the maximum wall temperature is lower than the quasi-critical temperature of kerosene, and the temperature rise can be seen from the central flow temperature trend. However, when the inlet oil temperature is $300 \mathrm{~K}$ or $400 \mathrm{~K}$, the oil temperature in the
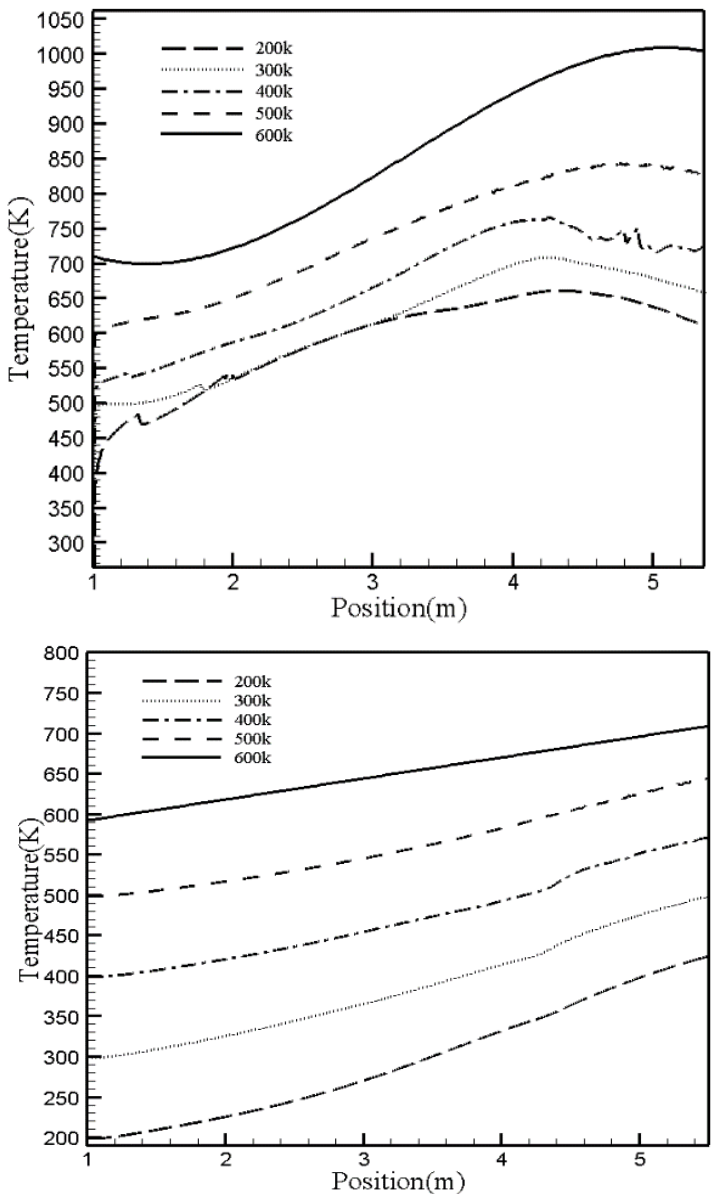

a. Wall temperature distribution along the axis b. Center flow oil temperature distribution along the axis

Figure 6. Axial changes of wall temperature and central flow oil temperature at different inlet temperatures.

Figure 7 shows the distribution of central flow oil pressure in the axial direction. It can be seen from the figure that the static pressure of kerosene in the pipe gradually decreases along the flow direction. Under different inlet temperature conditions, the higher the inlet temperature, the larger the central flow oil pressure The greater the flow resistance, the greater the pressure loss. This is because under the near-wall area is critical at this time. Near the temperature, the physical properties change very drastically, and the oil temperature at the central flow also fluctuates at $x=4 \mathrm{~m}$. When the inlet temperature increases to $500 \mathrm{~K} 、 600 \mathrm{~K}$, the temperature of the wall surface exceeds the critical temperature of kerosene, and the temperature of the central flow changes. And smooth

condition that the inlet mass flow rate is constant, the higher the inlet temperature is, the smaller the kerosene density is, the larger the inlet speed is, and as the flow resistance increases, the pressure loss becomes larger.Through the analysis above, we can know that when the wall temperature is higher or lower than the critical temperature of kerosene, the physical parameters of kerosene do not change much, and no heat transfer deterioration occurs. When the wall temperature is near the critical temperature, the physical parameters of kerosene in the near-wall region change drastically. The heat transfer of kerosene in a circular tube will undergo three stages: normal heat transfer, heat transfer deterioration, and heat transfer enhancement.

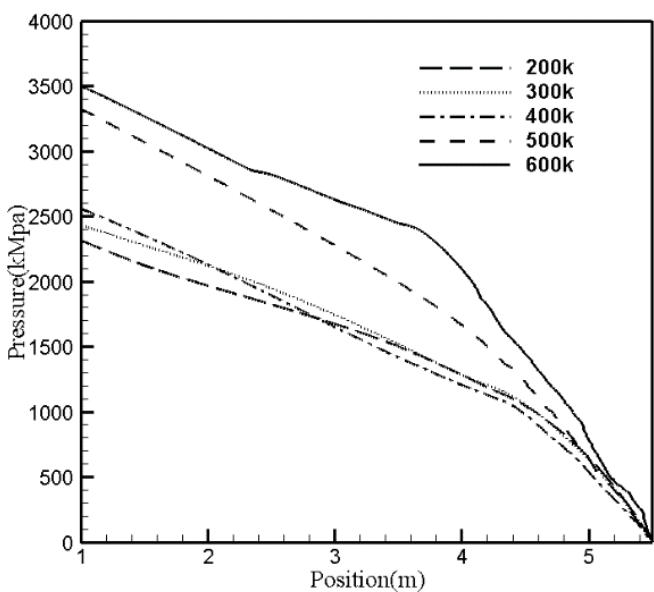

Figure 7. Variation of central flow oil pressure in the axial direction at different inlet temperatures.

\subsection{Heat transfer characteristics at different inlet pressures}

In order to study the heat transfer of aviation kerosene in a circular tube at different inlet pressures, the inlet pressure was changed from $2 \mathrm{MPa}$ to $10 \mathrm{MPa}$ at an inlet temperature of $300 \mathrm{~K}$, an inlet mass flow of $0.1 \mathrm{~kg} / \mathrm{s}$, and a heat flux density of $1 \mathrm{MW} / \mathrm{m}^{2}$. The specific entry parameters are shown in Table 2.

Table 2. Various operating conditions at different inlet pressures

\begin{tabular}{c|c|c|c|c|c}
\hline \multirow{2}{*}{$\mathrm{P}(\mathrm{MPa})$} & $\mathbf{1} \mathbf{k}(\mathrm{kg} / \mathrm{s})$ & $\mathrm{T}(\mathrm{K})$ & $\mathrm{Q}\left(M W / \mathrm{m}^{2}\right)$ & $\operatorname{Re}$ & $\rho\left(\mathrm{kg} / \mathrm{m}^{3}\right)$ \\
\hline 2 & 0.1 & 300 & 1 & 8848.28 & 789.68 \\
4 & 0.1 & 300 & 1 & 8841.94 & 791.56 \\
6 & 0.1 & 300 & 1 & 8836.11 & 792.07 \\
8 & 0.1 & 300 & 1 & 8835.23 & 794.21 \\
10 & 0.1 & 300 & 1 & 8835.01 & 796.04 \\
\hline
\end{tabular}



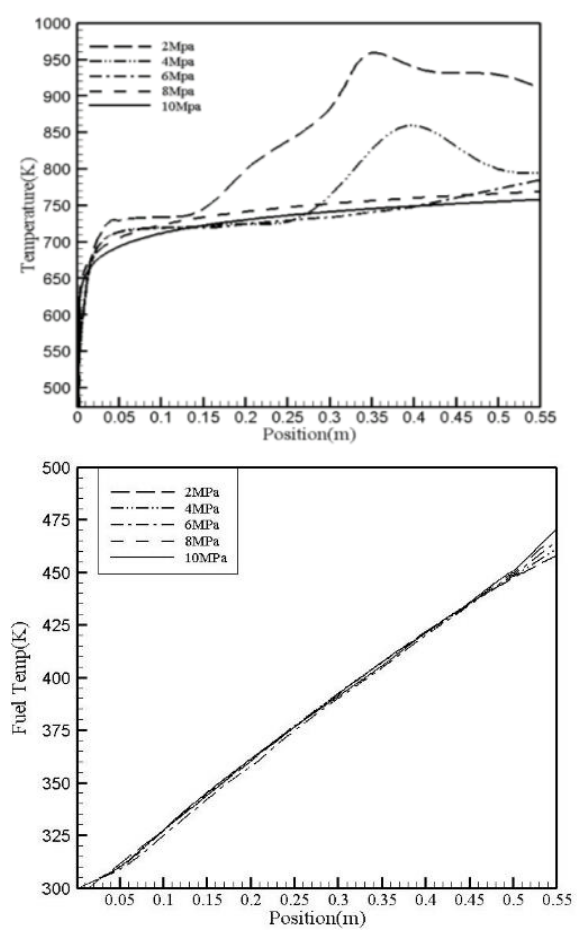

a. Wall temperature distribution along the axis oil temperature distribution along the axis

Figure 8. Axial distribution of tube wall temperature and central flow oil temperature at different pressures

Although the inlet pressure has a certain effect on the density and dynamic viscosity of kerosene, it has little effect and has a small effect on the inlet Reynolds number. Figure 8 shows the change of the wall temperature and the average oil temperature of kerosene in the tube under different pressures. It can be seen from the figure that, due to the influence of the wall heat flow density, on the one hand, the temperature gradually increases in the axial direction in the tube; on the other hand, the temperature in the near-wall region changes greatly. The results in Figure
8 (b) show that the average temperature of the kerosene in the tube basically overlaps under different pressures, and increases slightly with the increase of the pressure, which indicates that under the supercritical state of the kerosene in the tube, different pressures have little effect on the average temperature.

It can be seen from Fig. 8 (a) that the wall surface temperature changes at the inlet pressures of $2 \mathrm{MPa}, 4 \mathrm{MPa}$ and $6 \mathrm{MPa}$, but this phenomenon does not occur at high pressures of $8 \mathrm{MPa}$ and $10 \mathrm{MPa}$. This is mainly due to the The sudden change in position occurred according to heat, that is, the wall temperature suddenly increased, and then gradually decreased. When there is no heat transfer deterioration, the wall temperature is basically the same, but after the heat transfer deterioration occurs, as the inlet pressure increases, the wall temperature decreases, the location of the abnormality gradually moves towards the outlet, and the wall temperature changes. The smaller the amplitude, the lower the maximum temperature, which means that the increase in pressure helps to enhance heat transfer. As the inlet pressure continues to increase, the corresponding quasi-critical temperature increases, the temperature of the fluid does not reach the quasi-critical temperature, and heat transfer degradation is not easy to occur. Figure 9 shows the temperature distribution cloud diagram of kerosene in the pipe under different pressure inlet conditions. It can also be seen from the temperature distribution cloud diagram that the temperature distribution of kerosene in the pipe is basically the same in the inlet section, but the temperature of the outlet section increases with the pressure in the outlet section. It has been increased. Comparing these five figures, it can be seen that with the increase of the inlet pressure, the heat transfer effect from the wall surface to the axis gradually increases. The kerosene flowing in the tube can take more heat from the wall surface. The increase in pressure helps to enhance the heat transfer effect.

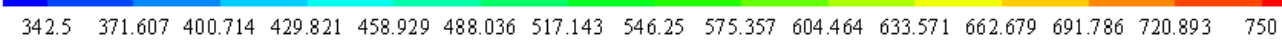
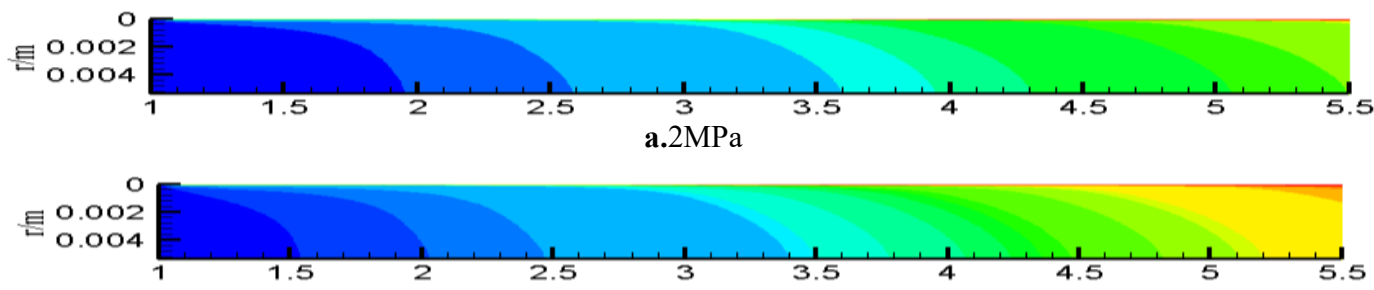

b. $4 \mathrm{MPa}$
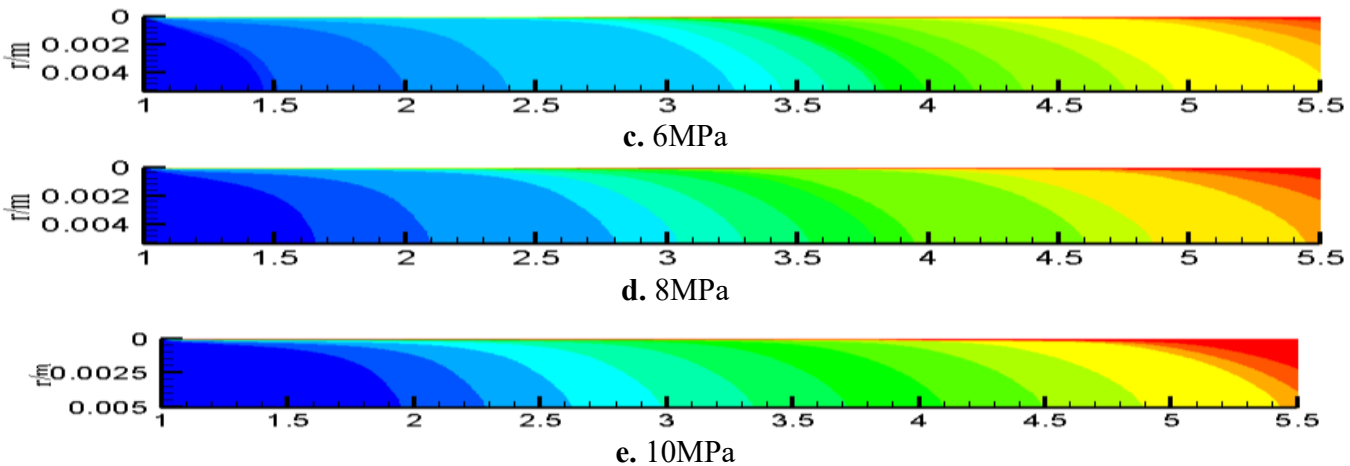

Figure 9. Temperature distribution of kerosene in the tube under different pressure inlet conditions. 


\section{Summary}

This chapter studies the flow and heat transfer characteristics of aviation kerosene in a circular tube. Firstly, the flow and heat transfer characteristics of supercritical kerosene were studied under different heat flow density distributions, and the following conclusions were drawn:

(1) With the increase of the inlet mass flow, the wall temperature and the central flow oil temperature gradually decrease, and the pressure loss will increase. The phenomenon of heat transfer deterioration occurs near the quasi-critical temperature of kerosene. The temperature change corresponding to the heat transfer deterioration at different mass flow rates is small, all in the range of $650 \mathrm{~K}$ $720 \mathrm{~K}$, which is near the quasi-critical temperature of kerosene.

(2) The inlet temperature also has a large effect on the flow and heat exchange of kerosene in the tube. The wall surface temperature and the central flow oil temperature increase with the increase of the inlet temperature. When the wall surface temperature is higher or lower than the kerosene critical temperature, The physical parameters of kerosene do not change much, and there is no deterioration in heat transfer. When the wall temperature is near the critical temperature, the physical parameters of kerosene in the near-wall region change drastically. The heat transfer of kerosene in a circular tube will undergo three stages: normal heat transfer, heat transfer deterioration, and heat transfer enhancement.

(3) Under the condition of constant heat flow density, the heat transfer characteristics of kerosene with different inlet pressure in the circular tube were analyzed. It is found that the heat transfer deterioration occurs near the quasi-critical temperature due to the drastic changes in physical properties. As the pressure increases, the heat transfer deterioration weakens, and increasing the pressure can improve the convective heat transfer effect.

\section{References}

1. Xiao Hongyu. Analysis and Design of Reinforced Active Cooling Structure Reinforcement for Supercombustion Stamping Engine [D]. Xi'an: Master's Thesis of Air Force Engineering University, 2007

2. Wang Yingjie. Review of heat transfer characteristics of supercritical fluids [J]. Science \& Technology Review, 2012, 30 (33); 74-79

3. Li Xunfeng, Zhong Fengquan. Numerical study of flow and heat transfer of aviation kerosene tube under supercritical pressure. [J] Propulsion Technology. 2010.Vol.31.No.4

4. Xianliang Lei, Huixiong Li, Shuiqing Yu, Tingkuan Chen. Numerical investigation on the mixed convection and heat transfer of supercritical water in horizontal tubes in the large specific heat region [J]. Computers and Fluids. 2012, 64, 127-140

5. Dong Eok Kim, Moo Hwan Kim. Two layer heat transfer model for supercritical fluid flow in a vertical tube. [J]. The Journal of Supercritical Fluids. 2011, 58 (1), 15-25

6. Fan, X J;Yu,G;Li,J G;Lu,X N;Sung. Performan -ce of Supersonic Model Combustors with Distributed Injection of Supercritical Kerosene [R]. AIAA 2007 5406, 2007

7. Gong, Les. Real-Time Simulation of Aeroheating of the Hyper-X Airplane [R] NASA. 20110014692, 2011

8. Guo Chaobang, Li Wenjie, Xing Ya. Research progress on active cooling and high temperature resistant structural components of French supercombustion stamping engines. Aeronautical Missiles, 2011 (11)

9. Ungar, Eugene K .; Schunk, Richard G: Spacecraft Radiator Freeze Protection Using a Regenerative Heat Exchanger [R] NASA 20110012266,2011

10. Moholt, Matthew. ARMD Hypersonics Project Materials and Structures: Testing of Scramjet Thermal Protection System Concepts [R]. NASA. 20090012145, 2009 